\title{
Erratum: "New exact analytical results for two quasiparticle excitation in the fractional quantum Hall effect" [Condens. Matter Phys., 2019, 22, No 2, 23703: 1-9, doi:10.5488/CMP.22.23703]
}

\author{
Z. Bentalha \\ University of Tlemcen, Theoretical Physics Laboratory, 13000 Tlemcen, Algeria \\ Received October 6, 2019
}

A forgotten reordering type error in coding the two-quasiparticle $\mathrm{CF}$ wave function of equation (5.3) leads to insignificant errors for the found results of order $\left(\sim 10^{-5}\right)$, the corrected results of table 5 are as follows:

Table 5. The two quasiparticle excitation energies (in units of $e^{2} / l_{0}$ ) are given for the $\mathrm{CF}$-wavefunction at the filling $v=\frac{1}{3}$ for systems with up to $N=7$ electrons.

\begin{tabular}{lllllll}
\hline \hline$N$ & & & & & \\
& $\bar{\varepsilon}_{\mathrm{bb}}$ & $\bar{\varepsilon}_{2 \mathrm{ebCF}}^{\mathrm{qp}}$ & $\bar{\varepsilon}_{2 \mathrm{eeCF}}^{\mathrm{qp}}$ & $\bar{\varepsilon}_{2 \mathrm{CF}}^{\mathrm{pp}}\left(S_{0}\right)$ & $\varepsilon\left(S_{0}\right)$ & $\varepsilon_{2 \mathrm{CF}}^{-}$ \\
\hline 4 & 0.693064 & -1.439563 & 0.421445 & -0.182232 & -0.388855 & 0.206623 \\
5 & 0.774869 & -1.602266 & 0.486072 & -0.214759 & -0.390255 & 0.175496 \\
6 & 0.848826 & -1.748533 & 0.548659 & -0.236691 & -0.391517 & 0.154826 \\
7 & 0.916837 & -1.882486 & 0.609371 & -0.251488 & -0.392624 & 0.141136 \\
\hline
\end{tabular}

It should be emphasized that these insignificant errors have no impact on the subsequent analysis made in this paper.

\author{
Erratum: “Нові точні аналітичні результати для збудження \\ двох квазічастинок при дробовому квантовому ефекті Гола" \\ [Condens. Matter Phys., 2019, 22, No 2, 23703: 1-9, \\ doi:10.5488/CMP.22.23703]
}

3. Бентала

Університет Тлемсена, лабораторія теоретичної фізики, 13000 Тлемсен, Алжир 


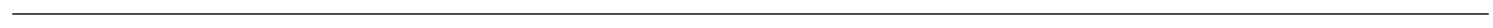

